\title{
COVID-19 Pandemic and Uptake in Suicide Attempt Among Young People of Minority Population: A Case Series
}

\author{
Chiedozie Ojimba ${ }^{\mathrm{a}, \mathrm{c}}$, Terrence Tumenta ${ }^{\mathrm{a}}$, Amod Thanju ${ }^{\mathrm{a}}$, Kenneth Oforeh ${ }^{\mathrm{a}}$, \\ Joy Osaji ${ }^{\mathrm{b}}$, Amal Saha ${ }^{\mathrm{a}}$, Leon Valbrun ${ }^{\mathrm{a}}$
}

\begin{abstract}
The first case of coronavirus disease 2019 (COVID-19) was reported in Wuhan China on December 31, 2019. COVID-19 was declared a global pandemic on March 11, 2020. To reduce the spread of this virus, the World Health Organization (WHO) and the Center for Disease Control (CDC) recommended self and mandatory quarantine of exposed individuals and self-isolation. However, the psychological impact of this pandemic includes new onset or worsening of existing mental illnesses which include but are not limited to anxiety, depression from social isolation, eating disorders, and uptake in suicidality either in isolation or part of mental illness symptomatology. In the USA, suicide is the second leading cause of death among people aged 10 - 34 years while globally, it is the second cause of death among people aged 15 - 29 years. The authors present a case of two young women of minority population with no prior psychiatric illnesses who presented to the psychiatry emergency room with suicidal attempts due to COVID-19 pandemic-related psychosocial stressors.
\end{abstract}

Keywords: COVID-19; Suicidality; Mental illness; Young people; Minority population; Psychosocial stressors; Social determinants of health

\section{Introduction}

The virus causing the coronavirus disease 2019 (COVID-19) belongs to the coronavirus family, which is a RNA virus with seven strains known to cause disease in humans [1]. This virus causes different constellations of symptoms like fever, cough, shortness of breath or difficulty breathing, fatigue, headaches, the new loss of taste and smell, sore throat, nasal congestion nausea or vomiting, and diarrhea [2]. The worst symptom is severe respiratory distress, causing hypoxia and requiring the

Manuscript submitted September 25, 2020, accepted October 3, 2020

Published online October 21, 2020

Interfaith Medical Center, Brooklyn, NY, USA

${ }^{\mathrm{b}}$ Texas Tech University Health Science Center, Lubbock, TX, USA

${ }^{c}$ Corresponding Author: Chiedozie Ojimba, Interfaith Medical Center, Brook-

lyn, NY 11213, USA. Email: cojimba@interfaithmedical.org

doi: https://doi.org/10.14740/jmc3594 use of mechanical ventilation, giving it the name severe acute respiratory syndrome coronavirus 2 (SARS-CoV-2) [1]. The mode of transmission of COVID-19 is through respiratory droplets and close person-to-person contact [1]. The first case of COVID-19 was reported in Wuhan, China on December 31, 2019 [1]. COVID-19 was declared a global pandemic on March 11, 2020 [1]. To reduce the spread of this virus, the World Health Organization (WHO) and the Center for Disease Control (CDC) recommended self and mandatory quarantine of exposed individuals and self-isolation $[3,4]$. These recommendations were implemented by many countries leading to the total lockdown of schools and most businesses. The isolation and self-quarantine reduced the rate of transmissions and death from the physical effects of the virus [5]. However, there were other stressors and devastating effects which arose from the self-quarantine which included but were not limited to economic and psychological impacts. The psychological impact of this pandemic includes new onset or worsening of existing anxiety from the fear of contracting the virus, financial and economic uncertainties, depression from social isolation, eating disorders, and uptake in suicidality [5, 6]. A study of Chinese high school adolescents by Zhou et al highlighted some of the challenges faced by adolescents as a result of the quarantine from COVID-19. They include an increase in family violence, mental illnesses like post-traumatic stress disorder (PTSD), anxiety disorders, depression, increased illicit substance use, grief, and more access to the internet and social media [5]. The study revealed a very high prevalence of depression $(43 \%)$ and anxiety $(37 \%)$ among adolescents in Wuhan, China. Women were most affected [5]. These factors may have a significant impact on suicidality.

Suicide is defined as death caused by self, where the individual had every intent to die [6]. Failed suicidal is an attempt when the individual intended to die from the actions taken but failed [6]. Suicide is a public health priority, as about 800,000 people globally commit suicide annually. The rate of suicide attempts continues to increase annually. Suicide is the third leading cause of death among people of $15-19$ years, and the second cause of death among people of $15-29$ years [7]. It means that the incidence and prevalence of suicide among young people are very high. According to the United Nations (UN), young people are those individuals aged $10-24$ years.

In the USA, suicide is the 10th leading cause of death and the second leading cause of death among people of $10-34$ years [8]. The risk for suicide includes depression, previous suicide attempts, substance use disorders (SUDs), person- 
ality disorders, impulsivity due to the immaturity of young people's prefrontal cortex, and stressful life events. Stressful life events among this age group include family conflicts, academic stressors, temperament, and trauma $[9,10]$. The selfisolation and pandemic lockdown were implemented to prevent the spread of the virus which may have led to stressful life events. These stressful life events may increase the rate of suicide uptake in the face of the COVID-19 pandemic [5]. We report a case of two young people of minority population with no prior psychiatric illnesses who presented to the psychiatry emergency room 4 days interval apart with suicidal attempts due to COVID-19 pandemic-related psychosocial stressors.

\section{Case Reports}

\section{Case 1}

The patient is a 23-year-old African American female student, single, and domiciled with family, with no reported prior past psychiatric history and past medical history who was brought to the psychiatric emergency room of a sister hospital by the emergency medical services (EMSs) in the context of suicidal attempt after she swallowed 20 pills of sitagliptin-metformin (Janumet). After the initial primary workup of hypoglycemia and other medical complications, the patient was transferred to the Interfaith Medical Center psychiatric emergency room for further psychiatric evaluation and admission.

Upon initial evaluation, she reported that she was in her usual state of health until 1 day before presentation when she started feeling overwhelmed and overrun by life and being frustrated with coronavirus quarantine, doing the same thing over and over for months, tired of getting up every day, eating and sitting at home. The patient reported low energy, hopelessness about her life, poor sleep, sleeping $2 \mathrm{~h}$ per night with early morning awakening of 1 -month duration. The patient stated that she and her twin sister planned to kill themselves, and both of them took about 20 pills of Janumet. Patient reported excessive worrying since the coronavirus pandemic and stated that life of being in the house with five people, not doing anything, not in a romantic relationship was not worth living. The patient reported smoking marijuana with her twin sister on the day she overdosed on Janumet pills. She identified her main stressors as not being able to go to Manhattan daily as has always been done before the COVID-19 pandemic lockdown in New York City. The patient reported studying Fashion and Arts and missing her school, nature experiences, and city life, which are heavily weighing on her. The patient denied any suicidal ideation or attempt in the past, a family history of suicidality, and any history of psychiatric illness or in the family. She also denied any history of psychological trauma before the pandemic lockdown. Per collateral was obtained from the patient's mother who reported that the patient was usually outgoing with liking for movie theaters. The mother reported that the patient has been isolated at home and stressed-out due to coronavirus. She corroborated the patient's story of no previous suicidal attempt, psychiatric illness, history of childhood trauma, a complication in pregnancy or childbirth, and no fam- ily history of mental illness.

On initial investigations, the COVID polymerase chain reaction (PCR) test was negative, and the chest X-ray finding was clear lung fields with no infiltrates. Electrocardiogram (EKG) showed normal sinus rhythm with QTc as $431 \mathrm{~ms}$. Urine toxicology was positive for cannabinoids and thyroid-stimulating hormone (TSH) was $6.750 \mu \mathrm{IU} / \mathrm{mL}$. The potassium level was $3.4 \mathrm{mmol} / \mathrm{L}$ during admission which was below the expected range, it was supplemented and a repeat 9 days after was 4.5 $\mathrm{mmol} / \mathrm{L}$. There were no other pertinent findings as complete blood count, complete metabolic panel, urinalysis, and daily blood glucose checks were all normal. She was started on Zoloft $50 \mathrm{mg}$ daily for depression and trazodone $50 \mathrm{mg}$ HS for sleep. Patient was placed on 1:1 constant observation for being actively suicidal and safety reasons.

On the first day in the inpatient unit, she was agitated, demanding to be discharged and exhibiting poor impulse control, insight, and impaired judgment with maladaptive coping skills. Patient refused to take Zoloft medication stating she was not depressed. On the second day, the patient continued to be agitated, demanding to be discharged and exhibiting poor impulse control/insight and impaired judgment. The patient was offered group and individual therapy; however, she refused. On day 3, she took Zoloft $50 \mathrm{mg}$ and complained of nausea. The dose of Zoloft was reduced to $25 \mathrm{mg}$ due to gastrointestinal (GI) side effects. From day 4 to day 6 , the patient continued to refuse medication and remained minimally cooperative with the treatment team, and continued to exhibit poor impulse, insight, and judgment. She continued to request for discharge stating that she was not depressed but was only overwhelmed by the COVID-19 pandemic situation and did not need medication. On day 7 , the patient became minimally cooperative, became more engaged with the treatment team, and started coming for individual and group therapy sessions. Milieu and art therapy were provided in the unit. During her individual-supportive therapy, she was taught the basic coping skills to deal with difficult life stressors. Patient was observed to be engaging with peers, participated in individual-supportive therapy, and identifying healthy coping strategies. She was observed to be well engaged in art therapy sessions, and participated in coloring, drawing, writing, and playing puzzles. Her impulse control and judgment became fair and she continued to engage with the treatment team. Based on her clinical improvement, the treatment team decided to discontinue 1:1 constant observation. On days 8 and 9 , the patient continued to show improvement in her behavior, impulse control, verbalized understanding of coping strategies she needs during stressful situations. By day 10, she showed good impulse control, well behaved on the unit, and exhibited good insight and judgment. The patient denied suicidal ideation, intent, or plan and was regretful and remorseful of her actions. She was discharged after the treatment team ascertained that she was no longer a danger to herself. Before her discharge, the treatment team had a family meeting with her mother, and psychoeducation was provided on the importance of compliance with aftercare appointments. The patient's diagnosis was revised from major depressive disorder to adjustment disorder with mixed anxiety and depressed mood. She was discharged home with outpatient treatment plans. The patient was evaluated by the medical 
team daily throughout her 10 days of inpatient hospital course. The patient was supplemented with potassium due to low level on admission and she was referred to the outpatient endocrine clinic for subclinical hypothyroidism and repeat of TSH and free T4 within 2 weeks of discharge.

\section{Case 2}

The patient is a 25-year-old African American woman, single, unemployed, financially supported by government assistance, and domiciled alone with her 3-year-old boy in an apartment complex, with no reported prior psychiatric history and past medical history. She was brought into the psychiatric emergency room by ambulance activated by her boyfriend for a suicidal attempt.

Upon evaluation in the psychiatric emergency room, she reported feeling depressed, anhedonic, hopeless, helpless, and worthless for 4-week duration. She also reported poor sleep for 6 months; she reported that her sleep was interrupted by early morning awakenings since February 2020. The patient reported that she had always been depressed for the past 3 years although she has been able to function and there was no official diagnosis by a psychiatrist, or any other health provider; she has never taken antidepressant medication. She reported that her depressive symptoms became aggravated when she lost her job in March 2020 due to the COVID-19 pandemic and had not been able to find another job since then. She reported a psychosocial stressor of not being able to support herself and her son financially. She also reported a lack of financial support from her son's father and family. She reported that minutes before attempting suicide which she did by drinking cleaning detergent, she had attempted calling her stepmother and her dad but none of them answered. She reported that she felt nobody cared for her, and she felt the need to commit suicide by jumping from the roof of a building, but her boyfriend interrupted, and she ran into her room, locked the door, and drank the cleaning detergent, Fabuloso. However, she was interrupted by her boyfriend. The patient denied a prior history of suicidal ideation, intent, plan, or attempts. She also denied any family history of suicidality or mental illness. Blood investigation included complete blood count, the complete metabolic panel was done on admission, and values were within normal limits. Her urine toxicology screen was positive for cannabinoids. EKG showed normal sinus rhythm and QTc was $397 \mathrm{~ms}$. The patient's COVID-19 test was negative and chest X-ray imaging was also negative for any infiltrates. Urinalysis was negative for urinary tract infection and the coagulation panel was within normal limits. She was medically cleared. The patient was admitted to the psychiatric inpatient unit for stabilization and safety. She was placed on 1:1 constant observation, started on escitalopram 10 mg orally daily for her depressive symptoms and trazodone 50 $\mathrm{mg}$ at bedtime for sleep.

In the inpatient unit, she was screened for depression with the Hamilton Depression Rating Scale (HDRS) for which she scored 18. She was also screened for bipolar disorder with the Mood Disorder Questionnaire which did not reveal any symptoms of bipolarity. The treatment team continued her on escit- alopram $10 \mathrm{mg}$ orally daily, trazodone $50 \mathrm{mg}$ orally at bedtime, and 1:1 constant observation for safety. Although she tolerated these medications with no reported side effects, the patient continued to endorse depressed mood, suicidal ideation, and anhedonia. Individual supportive therapy, group therapy, art therapy, and milieu therapy were started. Her 1:1 constant observation was discontinued on day 3 of admission because the patient was no longer endorsing suicidal ideations, intents, or plans. She reported feeling safe while in the inpatient unit. She became engaged and less isolated. She actively participated in different therapy sessions in the unit and continued to remain compliant with her medications.

On day 7 of the patient's admission, her interaction with others in the unit continued to improve, and she was more engaging and compliant with treatment plans. She was becoming more visible in the unit. However, she continued to exhibit poor coping skills and needed improvement in her maladaptive coping skills. The patient continued to be compliant with her medication. On day 8, the repeat EKG was done because of the synergistic effect of trazodone and escitalopram on QTc and the interval was $412 \mathrm{~ms}$.

The patient continued to show significant clinical improvement. On day 11, her depressive symptoms had significantly improved, and her affect was brighter. She continued to remain an active participant during the group therapy sessions and followed all group directives. She had made a lot of friends while in the unit and became more goal- and futureoriented. On day 12, repeat screening with HDRS was negative for depressive symptoms, and the score was zero. She had learned some basic coping skills while in the unit and was motivated to continue with outpatient aftercare treatment plans. The patient was discharged home on day 13. It is worth noting that the patient was closely monitored by the medical team during her stay, and no medical diagnosis was provided, or complications found.

\section{Discussion}

Our case reports show two young adults with no existing medical or psychiatric history, who attempted suicide impulsively. The two share the same psychosocial stressors: unemployment and social isolation in the context of the COVID-19 crisis. Additionally, they may have been experiencing ongoing stress with the fear of the unknown, fear of death, family and friends potentially dying from COVID-19, and increased close contact with one's living companions, with an increase in the potential for arguments and conflicts. These two individuals share the same demographics, African American women, and presented to the psychiatric emergency room 4 days interval apart in June 2020 reflecting an unusual incidence and presentation of suicidality cases in our hospital especially among young people.

With the outbreak of this pandemic, and foreseeing an uptake in suicide risks, the WHO and other agencies published recommendations on mental health and psychosocial considerations during the COVID-19 outbreak and the psychological effects of quarantine [11, 12]. A major impact of this pandemic was an increase in the rate of unemployment. Kawohl and Nordt had reported two scenarios in which the 
COVID-19 pandemic and unemployment could affect suicide. In the high scenario, the worldwide unemployment rate would increase from $4.936 \%$ to $5.644 \%$, which would be associated with an increase in suicides of about 9,570 per year. In the low scenario, unemployment would increase to $5.088 \%$, associated with an increase of about 2,135 suicides [13]. Earlier in March 2020, Klomek reported that during and following the COVID-19 outbreak and the outcomes of isolation and quarantine, we might see an increase in suicide ideation and behavior among at-risk populations [14]. This was corroborated by Conejero et al who reported that social distancing and quarantine could increase the feeling of disconnection and the perception of social pain in vulnerable individuals [15]. These current narratives are similar to those reported in the past. The Veterans' Affairs, in the paper, From Science to Practice: The Effect of Unemployment on Suicide Risk, found that in the USA, unemployment poses a greater risk for suicide with a person's increasing age. With longer periods of unemployment, the risk of suicide increases and peaks with the first 5 years [16]. In the early 2000s in New Zealand, Blakely et al counted deaths by suicide in their population within 3 years of their national census. Unemployed people were 2 - 3 times more likely to commit suicide than those who were employed at the time of the census [17]. This trend was contrary to that reported by Kim and Cho in 2017 with their study which showed that unstable employment or low level of employment protection showed an increased effect on suicide rates, whereas unemployment itself showed no difference [18]. Following the great recession of 2008, some studies reported that long-term ( $>52$ weeks) unemployment was significantly correlated with large negative effects on mental health [19]; that there were estimated 4,750 excess suicide deaths in the USA after the recession over the 3 years following it [20]; and that a 10\% increase in unemployment was significantly associated with the parallel $1.4 \%$ increase in male suicides [21].

The way our patients attempted self-harm shows how impulsive they were. There have been many studies showing the link between impulsivity and suicide in adolescents and young adults. McHugh et al conducted a systematic review and reported that multiple facets of impulsivity are associated with suicidal behavior in young people [22]. In their study, Liu et al stated that their systematic review provided general support for self-injurious thoughts and behaviors being associated with greater impulsivity, as conceptualized within a behavioral and cognitive neuroscience perspective [23]. This may have to do with the development of the prefrontal cortex in adolescents and young adults.

Social distancing from real contact with family and friends likely diminishes the protection from suicide that is usually enjoyed by social connections. Given that both patients were quarantined at home during the pandemic, it could be that their use of the internet and social media increased significantly, though this was not explored. Marchant et al found that the relationship between internet use and self-harm/suicidal behavior was particularly associated with internet addiction, high levels of internet use, and websites with self-harm or suicide content. They concluded that young people appear to be increasingly using social media to communicate distress, particularly to peers [24]. This may be a possible explanation for their actions despite of the lack of prior psychiatric or medical history in both of our patients.

Lastly, our patients are African American women. Fitzpatrick et al recently published the results of an online national survey of 10,368 US adults in which they reported that Blacks, Hispanics, women, and those who are younger appeared to be acutely at risk for suicidality, even net of mitigating resources [25]. This is part of a bigger problem involving the social determinants of health that include but are not limited to access to healthcare, economic insecurity, poor neighborhood and housing conditions, and availability of resources. Turner-Musa et al reported that social determinants affecting minority populations are believed to make them more vulnerable to the coronavirus [26]. They stated that in the USA, Blacks, Hispanics/ Latina, and Native Americans are disproportionately impacted by COVID-19 [26]. Moore et al reported that the findings from their analysis aligned with other data indicating that black persons are overrepresented among COVID-19 cases, associated hospitalizations, and deaths in the USA [27]. Fitzpatrick et al conclude that it is particularly important to consider these social determinants when proposing prevention and intervention strategies that are designed to target those with the highest suicidality risk [25].

\section{Conclusions}

This case series presents some important findings that contribute to our understanding of the increasing mental health consequences of the COVID-19 pandemic in the USA, especially as it pertains to uptake in suicidality in minority groups. As more information is acquired about this disease and its ramifications on the mental health of the population, strategies for better prevention and intervention may be developed. It is incumbent on governments to learn from previous pandemics and recommended strategies to mitigate risks on minority ethnicities due to socioeconomic disadvantages while protecting the mental health of the entire population.

\section{Acknowledgments}

None to declare.

\section{Financial Disclosure}

None to declare.

\section{Conflict of Interest}

None to declare.

\section{Informed Consent}

\author{
Not applicable.
}




\section{Author Contributions}

$\mathrm{CO}$ contributed to the design of the study, case write-up, data collection, interpretation of the data, helped to write the final draft of the manuscript, and has full access to all the data in the study as the primary author of the manuscript. TT contributed to writing the Discussion. AT and $\mathrm{KO}$ contributed to writing the cases. JO contributed to writing the Introduction. AS and LV contributed to the design of the study and supervised the writing of the final draft of the manuscript.

\section{Data Availability}

The data supporting the findings of this study are available from the corresponding author upon reasonable request.

\section{References}

1. Cennimo DJ. August 20, 2020. Coronavirus disease 2019 (COVID-19): practice essentials, background, route of transmission. Medscape. https://emedicine.medscape. com/article/2500114-overview\#showall.

2. Coronavirus Disease 2019 (COVID-19) - Symptoms. May 13, 2020. Centers for Disease Control and Prevention. https:/www.cdc.gov/coronavirus/2019-ncov/symptoms-testing/symptoms.html.

3. Timeline: WHO's COVID-19 response. 2019. World Health Organization. https://www.who.int/emergencies/ diseases/novel-coronavirus-2019/interactive-timeline?g clid=Cj0KCQjwp4j6BRCRARIsAGq4yME1puwRDU mQnsiMAbKSq3f-LDb7EpzEEHem295QV51xJMVmanxKv4aAnCDEALw_wcB\#event-71.

4. COVID-19 Case Surveillance Public Use Data | Data | Centers for Disease Control and Prevention. August 1, 2020. Center for Disease Control and Prevention. https:// data.cdc.gov/Case-Surveillance/COVID-19-Case-Surveillance-Public-Use-Data/vbim-akqf.

5. Zhou SJ, Zhang LG, Wang LL, Guo ZC, Wang JQ, Chen JC, Liu M, et al. Prevalence and socio-demographic correlates of psychological health problems in Chinese adolescents during the outbreak of COVID-19. Eur Child Adolesc Psychiatry. 2020;29(6):749-758.

6. Sadock BJ, Sadock VA, Ruiz P. Kaplan and Sadock's synopsis of psychiatry: behavioral sciences/clinical psychiatry 11th, North American. Edition Lippincott Williams \& Wilkins. 2015.

7. World Health Organization: WHO. September 2, 2019. Suicide. World Health Organization. https://www.who. int/news-room/fact-sheets/detail/suicide.

8. Preventing Suicide /Violence Prevention | Injury Center | CDC. 2019. Center for Disease Control. https:// www.cdc.gov/violenceprevention/suicide/fastfact. html\#:\%7E:text=Suicide $\% 20$ is $\%$ 20death $\% 20$ caused $\% 20$ by,a $\% 20$ result $\% 20$ of $\% 20$ their $\% 20$ actions.

9. Carballo JJ, Llorente C, Kehrmann L, Flamarique I, Zuddas A, Purper-Ouakil D, Hoekstra PJ, et al. Psychosocial risk factors for suicidality in children and adolescents. Eur Child Adolesc Psychiatry. 2020;29(6):759-776.

10. Carpiniello B, Pinna F. The reciprocal relationship between suicidality and stigma. Front Psychiatry. 2017;8:35.

11. WHO Mental health and psychosocial considerations during COVID-19 outbreak. March 12, 2020. https:// www.who.int/docs/default-source/coronaviruse/mentalhealth-considerations.pdf.

12. Inter-Agency Standing Committee Interim briefing note addressing mental health and psychosocial aspects of COVID-19 outbreak (developed by the IASC's reference group on mental health and psychosocial support). March 17, 2020. https://interagencystandingcommittee.org/other/interim-briefing-note-addressing-mental-health-andpsychosocial-aspects-covid-19-outbreak.

13. Kawohl W, Nordt C. COVID-19, unemployment, and suicide. Lancet Psychiatry. 2020;7(5):389-390.

14. Klomek AB. Suicide prevention during the COVID-19 outbreak. Lancet Psychiatry. 2020;7(5):390.

15. Conejero I, Berrouiguet S, Ducasse D, Leboyer M, Jardon V, Olie E, Courtet P. [Suicidal behavior in light of COVID-19 outbreak: Clinical challenges and treatment perspectives]. Encephale. 2020;46(3S):S66-S72.

16. https://www.mentalhealth.va.gov/suicide_prevention/ docs/Literature_Review_FSTP_Unemployment_FINAL_508_8-19-2019.pdf.

17. Blakely TA, Collings SC, Atkinson J. Unemployment and suicide. Evidence for a causal association? J Epidemiol Community Health. 2003;57(8):594-600.

18. Kim C, Cho Y. Does unstable employment have an association with suicide rates among the young? Int J Environ Res Public Health. 2017;14(5):470.

19. https://www.apa.org/pi/ses/resources/indicator/2012/04/ unemployment.

20. Reeves A, Stuckler D, McKee M, Gunnell D, Chang SS, Basu S. Increase in state suicide rates in the USA during economic recession. Lancet. 2012;380(9856):1813-1814.

21. Barr B, Taylor-Robinson D, Scott-Samuel A, McKee M, Stuckler D. Suicides associated with the 2008-10 economic recession in England: time trend analysis. BMJ. 2012;345:e5142.

22. McHugh CM, Chun Lee RS, Hermens DF, Corderoy A, Large M, Hickie IB. Impulsivity in the self-harm and suicidal behavior of young people: a systematic review and meta-analysis. J Psychiatr Res. 2019;116:51-60.

23. Liu RT, Trout ZM, Hernandez EM, Cheek SM, Gerlus N. A behavioral and cognitive neuroscience perspective on impulsivity, suicide, and non-suicidal self-injury: Metaanalysis and recommendations for future research. Neurosci Biobehav Rev. 2017;83:440-450.

24. Marchant A, Hawton K, Stewart A, Montgomery P, Singaravelu V, Lloyd K, Purdy N, et al. A systematic review of the relationship between internet use, self-harm and suicidal behaviour in young people: The good, the bad and the unknown. PLoS One. 2017;12(8):e0181722.

25. Fitzpatrick KM, Harris C, Drawve G. How bad is it? Suicidality in the middle of the COVID-19 pandemic. Suicide Life Threat Behav. 2020.

26. Turner-Musa J, Ajayi O, Kemp L. Examining social de- 
terminants of health, stigma, and COVID-19 disparities. Healthcare (Basel). 2020;8(2).

27. Moore JT, Ricaldi JN, Rose CE, Fuld J, Parise M, Kang GJ, Driscoll AK, et al. Disparities in incidence of COV-
ID-19 among underrepresented racial/ethnic groups in counties identified as hotspots during June 5-18, 2020 - 22 States, February-June 2020. MMWR Morb Mortal Wkly Rep. 2020;69(33):1122-1126. 